\title{
Beam quality measurements with Shack-Hartmann wavefront sensor and M2-sensor: comparison of two methods
}

\author{
J.V.Sheldakova, A.V.Kudryashov, V.Y.Zavalova, T.Y.Cherezova* \\ Moscow State Open University, Adaptive Optics Laboratory \\ Sudostroitelnaya Str 18, Bld. 5, Moscow, Russia 115407 \\ *Department of Physics, Moscow State University, Vorobyovy Gory, Moscow, Russia 119899
}

\begin{abstract}
In both scientific and industrial laser beam applications is essential for users to know what could be expected from the laser beam. That is why analysis of the laser beam parameters is very important during laser use in various industrial and scientific applications. To describe the beam one can use a beam quality factor $M^{2}$ that characterizes the degree of imperfection of a laser beam.

There are many methods of beam quality determination. The most common way is to use a device based on techniques described in the International Standard ISO11146 "Test methods for laser beam parameters: Beam widths, divergence angle and beam propagation factor". For example we can use M2-sensor that we design and produce in our Lab.

The measurement of the beam quality factor according to ISO11146 is not a simple procedure and might take a long time. And for some applications fast beam quality determination is needed. Moreover sometimes it is not necessary to know the exact value of $M^{2}$, only estimation of $M^{2}$ is just needed. And for the beam quality estimation we suggest to use Shack-Hartmann wavefront sensor. With this sensor we can easily and fast measure the wavefront of the beam and then according to the wavefront calculate $M^{2}$.

The comparison of two sensors is presented. Advantages and disadvantages are pointed out.
\end{abstract}

Keywords: beam quality factor $\mathrm{M}^{2}$, Shack-Hartmann wavefront sensor, M2-sensor

\section{INTRODUCTION}

The measurement of $M^{2}$ parameter became very popular because its value quantitatively compares the characteristics of the real beam to those of a pure $\mathrm{TEM}_{00}$. For the Gaussian beam $M^{2}=1$. For any other $M^{2}>1$. The relationship between $M^{2}$, wavelength $\lambda$, near-field waist diameter $d_{0}$ and divergence angle $\theta$ is ${ }^{1}$ :

$$
M^{2}=\frac{\pi}{4 \lambda} d_{0} \theta \text {. }
$$

The divergence angle is determined as:

$$
\theta=\frac{d_{f}}{f},
$$

where $d_{f}$ is the beam diameter at the focal distance $f$ of the used lens. So to evaluate $M^{2}$ it is necessary to measure both far-field beam diameter (to determine divergence angle) and near-field one.

\section{DIRECT $\mathrm{M}^{2}$ MEASUREMENTS WITH M2-SENSOR}

Various methods of beam quality determination could be suggested ${ }^{1-4}$. To measure $M^{2}$ a special sensor based on wellknown techniques is usually used. The most common way is to use a device based on a technique described in the International Standard ISO11146 "Test methods for laser beam parameters: Beam widths, divergence angle and beam propagation factor" ${ }^{1}$. The algorithm of measurements proposed in ISO11146 is based on a fixed-position lens and 
multiple beam-width measurements made through the focal plane waist (Fig.1). At least 20 measurements have to be taken where half of them should be distributed within one Rayleigh length on either side of the beam waist and half of them should be distributed beyond two Rayleigh lengths from the beam waist. The $\mathrm{M}^{2}$-factor and divergence angle could be easily obtained from performing a hyperbolic fit to the measured beam diameters along the beam propagation axis:

$$
d_{i}^{2}=A+B z_{i}+C z_{i}^{2}
$$

where $d_{i}$ is the beam diameter at the location $z_{i}, A, B, C$ are the hyperbola parameters.

Then the beam propagation factor can be calculated using simple equations ${ }^{1}$ :

$$
M^{2}=\frac{\pi}{4 \lambda} \cdot \sqrt{A C-B^{2} / 4} \text {. }
$$

In our Lab we designed M2-sensor ${ }^{5}$ to measure $M^{2}$; the setup is very simple, it consists of a focusing element and CCD placed on a moving stage (Fig.2). The disadvantage of this device and this technique is that we have to do lots of measurements and some adjustment is needed every time we move CCD. So this procedure might take a long time. But for some applications fast beam quality determination is needed. Moreover sometimes it is not necessary to know the exact value of $M^{2}$, only estimation of $M^{2}$ is just needed. And for the beam quality estimation we suggest to use ShackHartmann wavefront sensor.



Fig. 1. Principle of $M^{2}$ measurements

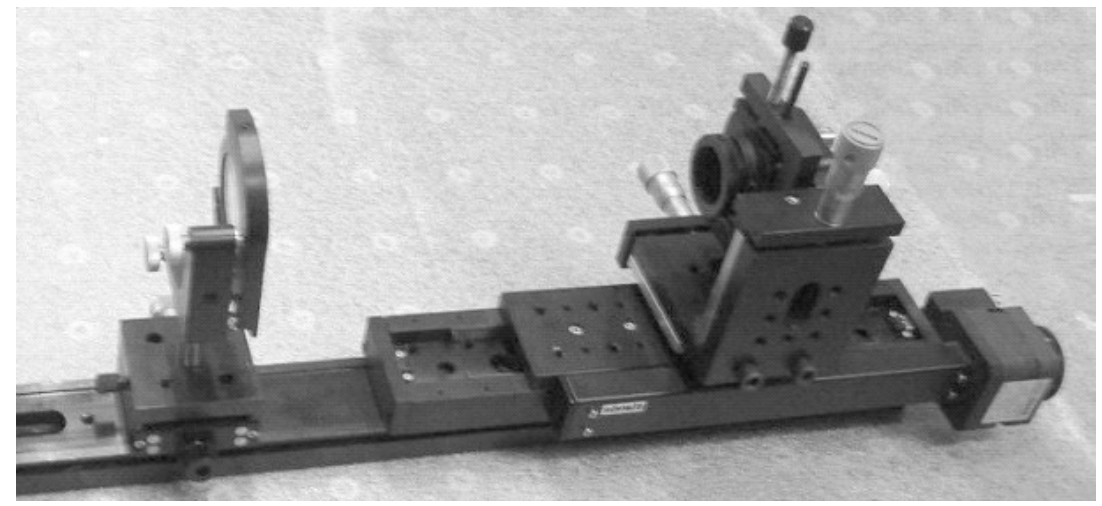

Fig. 2. Photography of our M2-sensor

\section{INDIRECT $\mathrm{M}^{2}$ MEASUREMENTS WITH SHACK-HARTMANN WAVEFRONT SENSOR}

With Shack-Hartmann wavefront sensor we can easily and fast measure the wavefront of the beam ${ }^{6}$. The wavefront determination by Shack-Hartmann wavefront sensor is based on the measurements of local slopes of a distorted wavefront $\partial \varphi / \partial n$ (Fig.3). So, the whole wavefront is divided in several subapertures by some phase plate or lenslet array and the deviation of the focal spot from some reference position in each subaperture is measured. Fig. 3 provides some idea about the work of the sensor. To measure a pattern of focal spots a standard CCD camera is used. We also produce in our Lab Shack-Hartmann Wavefront sensors (Fig.4). Beam resizing lens is used to match the diameter of input beam and CCD. 

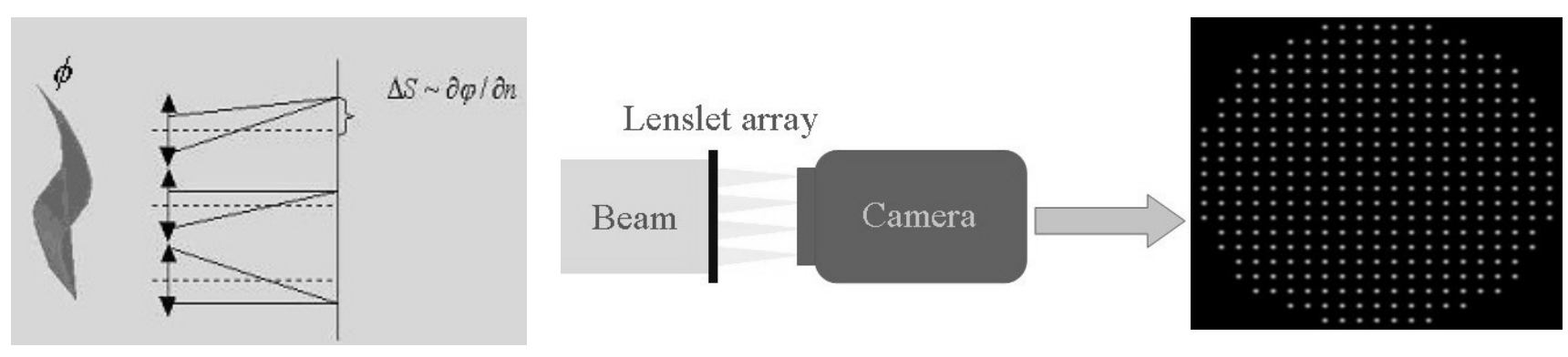

Fig. 3. Idea of Shack-Hartmann wavefront sensor



Fig. 4. Photography of our Shack-Hartmann wavefront sensor

Also from the CCD we have information about beam intensity. Of course we have only discrete intensity distribution and in case of huge ingomogenity of intensity distribution we cannot obtain correct result but still if the intensity is quite smooth we have enough amounts of points to reconstruct the intensity. And if beam phase and intensity distribution is known we can obtain far field intensity distribution $I_{\text {far field }}$ using FFT and then to calculate $M^{2}$ as

$$
M^{2}=\frac{d\left(I_{\text {far field }}\right)}{d_{\text {gauss }}} .
$$

The advantage of Shack-Hartmann wavefront sensor is that we need only one measurement to obtain $M^{2}$. So in comparison with M2-sensor we have less adjustment.

\section{EXAMPLES OF $\mathbf{M}^{2}$ MEASUREMENTS}

The results of our experiment made in Duisburg-Essen University, Germany (group of Alexander Tarasevitch), in femtosecond Ti:S laser are presented as an example of $M^{2}$ measurement. Here we had $10 \mathrm{~Hz}$ pulses with duration of $25 \mathrm{fs}$ and output energy of $200 \mathrm{~mJ}$.

First we measured wavefront with Shack-Hartmann wavefront sensor, P-V was around 1.1 (Fig.5a). Fig.5b shows far field intensity distribution calculated with FFT; for this distribution $M^{2}$ is 3 .

After that we placed 5 meter focusing lens in order to measure $M^{2}$ using standard procedure described in ${ }^{1}$. The focal plane intensity distribution is presented on Fig. 5 c. Obtained $M^{2}$ is also 3 in this case.

So in case of single mode beam we can successfully use Shack-Hartmann wavefront sensor to measure $M^{2}$. 

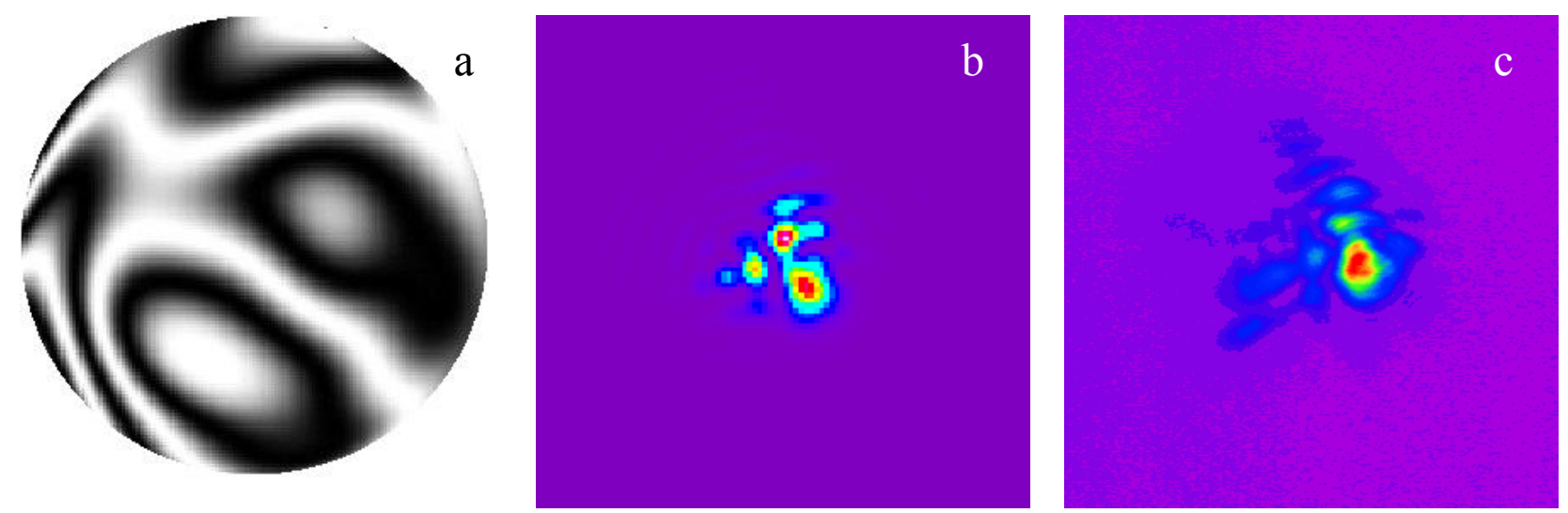

Fig. 5. Ti:S laser beam investigation. $\mathrm{a}$ - wavefront of the beam, $\mathrm{b}$ - calculated far field intensity distribution, $\mathrm{c}-$ measured far field intensity distribution

As a next example we present our experiment in DSO National Laboratories, Singapore (group of Lai King Seng). We tried to measure the aberrations of $\mathrm{CW} \mathrm{Nd:YAG} \mathrm{ceramic} \mathrm{laser} \mathrm{with} \mathrm{output} \mathrm{power} \mathrm{of} 500 \mathrm{~W}$. The output beam had a lot of high order modes.

First we measured wavefront (Fig.6a) of the beam and calculated far field intensity distribution. $M^{2}$ was around 3 in this case (Fig.6b). But as soon as we measured far field with $100 \mathrm{~mm}$ lens we found out that real $M^{2}$ is more than 55 (Fig.6c) and this value has nothing to do with the one measured with Shack-Hartmann wavefront sensor. The main reason of this awful result is that the beam was multi mode one. And as we cannot separate one mode from another we measured some averaged phase with Shack-Hartmann sensor. That is why the result might be incorrect, and the $M^{2}$ value is also unpredictable.
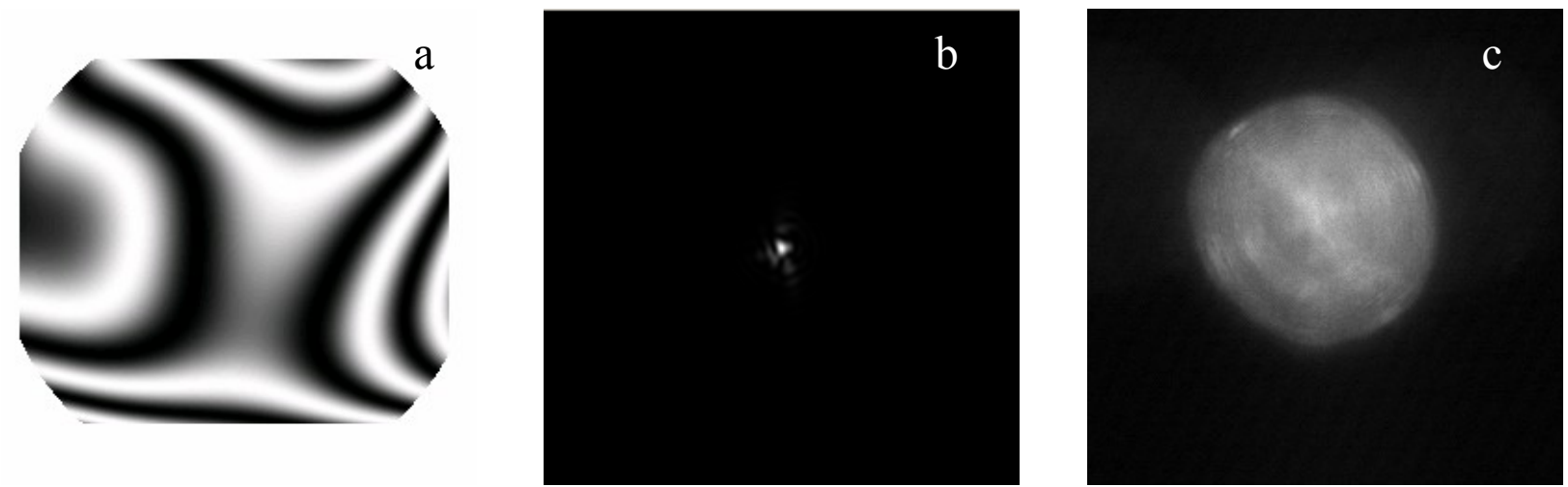

Fig. 6. Nd: YAG laser beam investigation. $\mathrm{a}$ - wavefront of the beam, $\mathrm{b}$ - calculated far field intensity distribution, $\mathrm{c}-$ measured far field intensity distribution

\section{COMPARISON OF TWO METHODS}

Table 1 shows the comparison of two sensors: M2-sensor and Shack-Hartmann wavefront sensor. The main advantage of Shack-Hartmann wavefront sensor is that we need only one measurement while using standard M2-sensor we have lots of work to do. Shack-Hartmann wavefront sensor is good for single mode and single pulse radiation. But unfortunately with this sensor we cannot obtain correct results for multimode radiation. And with M2-sensor of course we are able to measure any beam: single mode, multimode and $\mathrm{CW}$ or with high repetition rate. 
Table 1. Advantages and disadvantages of two sensors

\begin{tabular}{|c|c|c|}
\hline & M2-sensor & Shack-Hartmann wavefront sensor \\
\hline Advantages & $\begin{array}{l}\text { - Correct result while both single and } \\
\text { multimode beam analysis } \\
\text { - Convenient for CW or pulse lasers with high } \\
\text { repetition rate }\end{array}$ & $\begin{array}{ll}\text { - } & \text { Single measurement to obtain } M^{2} \text { value - } \\
\text { very fast and simple } \\
\text { - } & \text { Ability to analyse single mode radiation } \\
\text { - } & \text { Very convenient to measure single mode } \\
\text { single pulse laser radiation }\end{array}$ \\
\hline Disadvantages & - Slow measurements & $\begin{array}{l}\text { - Unpredictable result while multimode beam } \\
\text { analysis }\end{array}$ \\
\hline
\end{tabular}

\section{CONCLUSION}

The possibility of $M^{2}$ measurement with Shack-Hartmann wavefront sensor is shown. The comparison of M2-sensor and Shack-Hartmann wavefront sensor is presented. Advantages and disadvantages are pointed out. But we should be very careful while multi mode beams analysis.

Examples of both single-mode and multi-mode radiation analysis are presented. The possibility of Shack-Hartmann wavefront sensor use to measure $M^{2}$ of single-mode laser beam is shown. The result might be incorrect while multi-mode beam analysis.

\section{ACKNOLEGEMENTS}

Authors thank to Dr. Alexander Tarasevitch from Duisburg-Essen University, Germany, and Dr. Lai Kin Seng from DSO National Laboratories, Singapore, for the assistance in the experiment.

\section{REFERENCES}

1. Test method for laser beam parameters: Beam width, divergence angle and beam propagation factor, Document ISO/DIS 11146, International Organization for Standardization, 1996.

2. T. Jonston, "Beam propagation $\left(\mathrm{M}^{2}\right)$ measurement made as easy as it gets: the four-cuts method", Applied optics, 37(21), July 1998.

3. K. Roundy, "Propagation factor quantifies laser beam performance", Laser Focus World, 12, pp.119-122, 1999.

4. B. Eppich, S. Johansson, H. Laabs, H. Weber, "Measuring laser beam parameters, phase and spatial coherence using the Wigner distribution”, Proc. SPIE, 3930, pp. 76-86, 2000.

5. J.V.Sheldakova, T.Yu.Cherezova, A.Kudryashov, "Low-cost $\mathrm{M}^{2}$-sensor for the adaptive optical system", Proc. SPIE 4493, pp. 285-293, 2002.

6. V.Ye.Zavalova, A.V.Kudryashov, "Shack-Hartmann wavefront sensor for laser beam analysis", Proc. SPIE 4493, pp. 277-284, 2002. 